\title{
ADVANCES IN INFECTION EPIDEMIOLOGY AND CONTROL IN DENTAL HEALTHCARE SETTINGS
}

\author{
Stefano Petti ${ }^{1}$ \\ ${ }^{1}$ SA PIENZA UNIVERSITY, DEPARTMENT OF PUBLIC HEALTH AND INFECTIOUS DISEASES, ROME, ITALY
}

\begin{abstract}
Guidelines for the control of infection in dental healthcare settings became necessary since the eighties, following shocking events, such as the lethal outbreaks of hepatitis B among dental patients and the episode of the Floridian dentist who infected five patients with HIV. Guidelines were produced by the US Centres for Disease Control and Prevention and were periodically updated. Their success was and actually is remarkable, as demonstrated by the facts that they were adopted by many national professional organizations in the world and that, following their implementation, cases of infection transmission from dental healthcare workers (DHWs) to patients and vice versa reported from all over the world drastically decreased. Guidelines, initially based on the precautionary principle, are updated following the advances in scientific and technical research and are based on the assessment of the true risk for infection for DHWs and patients and on the design of effective control measures. Nevertheless, guidelines are far from being perfect, since many questions remained unanswered and many problems unresolved. The papers presented at the international workshop "Advances in Infection Epidemiology and Control in Dental Healthcare Settings" (February 9th 2013), organized by the Department of Public Health and Infectious Diseases of the Sapienza University of Rome (Italy). sought to bring some light in the shadowy areas of this field. There are four steps which lead to an effective infection control: the first of them is an efficient surveillance system of infections acquired in dental healthcare settings The second step is the specificity of scientific research. The third step is that guidelines must be applied by DHWs, who, therefore, must be involved in guideline design and in recommendation release. The fourth and final step is that infection control guidelines must be transnational.
\end{abstract}

Key words: dental healthcare setting, dentistry, infection control, guideline

\section{Introduction: why is infection control in dental healthcare settings necessary?}

Starting from the 80 's the Centers for Disease Control and Prevention of A tlanta (US) provided guidelines and recommendations for infection control in dental healthcare settings.
Guidelines became necessary because it was evident that dental therapy exposed patients and staff to the risk for serious infectious diseases, such as hepatitis B and C, HIV infection, tuberculosis. Indeed, the case of the dentist in Florida, who was HIV infected in 1986 and transmitted this infection to five patients and some of them died of AIDS, was shocking for the public and for healthcare workers from all over the world ${ }^{1}$. A nother astonishing example was the outbreaks of hepatitis B virus (HBV) transmission reported from US. In the late 70's one oral surgeon transmitted HBV infection to fifty-five patients ${ }^{2}$, while in the early 80 's a dentist transmitted it to nine patients ${ }^{3}$. The worrying aspects of these outbreaks were that dental healthcare workers were HBV carriers, but had no symptoms of hepatitis $B$, thus, they did not suspect to be sources of infection. In addition, case-fatality ratios in these outbreaks were high ( $>20 \%)$.

Guidelines were periodically updated according to the available scientific evidence and their success in terms of control of infectious diseases was indubitable. Although there are no field/clinical trials to support this assertion, the incidence of infectious diseases directly attributable to dental heal thcare workers and their low infection rates are a clearer support than the mere classical classification of the strength of scientific evidence, which requires doubleblind, randomized, controlled clinical trials ${ }^{4}$.

\author{
Address for correspondence: \\ Prof. Stefano Petti \\ Department of Public Health and Infectious Diseases \\ Sanarelli Building,Sapienza U niversity \\ P.le A Ido M oro 5,, I-00185 Rome, I taly \\ Phone/Fax: +3906 49914667 \\ E-mail: stefano.petti@uniromal.it
}

(C) 2013 Faculty of Medicine in Niš. Clinic of Dentistry in Niš. All rights reserved / (C) 2013 Medicinski fakultet Niš.

Klinika za stomatologiju Niš. Sva prava zadržana 
Guideline update is based on the assessment of the risk for infection for staff and patients -achievable through an efficient surveillance system; and on the design of effective preventive measures -built on the scientific evidence and not merely on the precautionary principle ${ }^{5}$.

On February 9th, 2013, the Department of Public Health and Infectious Diseases of the Sapienza U niversity of R ome (Italy), organized an international workshop entitled "A dvances in Infection Epidemiology and Control in Dental Heal thcare Settings", which reported the results of investigations and observational studies in the field of infection transmission and control in dental healthcare settings. These contributions sought to answer to specific unanswered questions.

\section{Where does microbial contamination of air of dental offices come from?}

The first paper sought to investigate the source of airborne microorganisms in the dental office. Airborne infectious diseases associated with human sources are influenza, meningitis, varicella, measles, mumps, various kinds of respiratory tract infections (RTIS), from pharyngitis to pneumonia, etc. These diseases are generally contagious and show typically high incidence. Infectious diseases associated with the biofilm of dental unit waterlines (DUWLs) are RTIs due to L egionella pneumphila, Pseudomonas aeruginosa, Mycobacterium, which are not or minimally contagious and affect immune-compromised individuals with consequently high case-fatality rates. This paper reported that the microorganisms from DUWLs were qualitatively and quantitatively different from the airborne microorganisms, thus suggesting that human microorganisms are likely to be the main source of airborne bacteria in the dental offices ${ }^{6}$. This result does not imply that DUWLs are not a source of airborne microorganisms, as it was previously demonstrated that more than $70 \%$ of DUWLs were contaminated by oral streptococci, selective microorganisms of the upper respiratory tract ${ }^{7}$, but only that human microorganisms prevail to bacteria from the aquatic biofilm.

\section{Is there a water quality index able to predict Legionella detection in dental unit waterlines?}

DUWLs are frequently colonized by legionellae, although detection rates vary between values as high as 30\% in hospitals and $0-4 \%$ in offices of general dental practitioners (GDPs). The question whether high detection rate is suggestive of a high risk for legionellosis among patients and staff is a matter of debate. If the risk for infection is estimated using the documented cases of infection, such a risk is probably minimal, since there are only two reports (one patient and one dental healthcare worker) of individuals who contracted legionellosis and in both cases, it was not ascertained whether the DUWL was the source of infection. If the risk is estimated using L egionella detection rate in DUWLs, such a risk is probably high. In absence of convincing scientific evidence, the precautionary principle could be invoked 5 and, therefore, it would be prudent to monitor water quality of DUWLs. But monitoring also is a problem, because L egionella investigation is long and expensive, therefore, surrogate markers of water quality, such as total cultivable heterotrophic flora could be more practical, if they are predictive of legionellae detection. This is the problem investigated by the second study, which was made in a dental hospital highly and persistently colonized by several Legionella species $^{8}$. In summertime, with Pseudomonas (L egionella competitor for the same ecological niche)- undetected, with total cultivable flora levels at $37^{\circ} \mathrm{C}$ and $22^{\circ} \mathrm{C}$ higher than 200 colony forming units (CFU)/mL (the threshold level proposed by the A merican Dental A ssociation), the probability that legionellae were detected in DUWLs was $29 \%$. A relatively high predictive value, but lower than expected since at that time legionellae were ubiquitous in the dental hospital.

\section{Are dental healthcare workers and students at risk for tuberculosis?}

The question whether DHWs are at risk for tuberculosis (TB) is controversial. Data from 
the literature suggest that among dentists/oral surgeons who work in hospitals, prisons, or endemic areas, prevalence of reactive tuberculin skin test (TST) is higher than in the general population, while it is normal among GDPs. Very interestingly, the fraction of false positives to TST among DHWs is extremely high. There are two explanations, the first is that for repeated TST occasions -many professional organizations suggest to make this test routinelyboosting (i.e., a T-cell response due to TSTs in absence of vaccination or M ycobacterium tuberculosis infection) may occur ${ }^{9}$. The second is that cross immunization between $M$. tuberculosis and other environmental mycobacteria, defined non-tubercular mycobacteria (NTM) isolated from water, such as Mycobacterium scrofulaceum or M ycobacterium intracellulare also may occur ${ }^{10}$ and DUWLs are often highly contaminated by NTM ${ }^{11}$. Despite this, prevalence of reactive TST among dental students $(17 \%)$ and DHWs $(20 \%)$ reported by the third study was high, compared with prevalence in the administrative staff $(7 \%)^{12}$. This high preval ence is even worrying considering that the selected subjects were not vaccinated against TB and that it was not due with the years of practice at the hospital.

\section{Is there a risk for transmission of Streptococcus pneumoniae in dentistry?}

Streptococcus pneumoniae is the major cause of death for pneumonia in the world, allegedly responsible for 826,000 deaths annually, particularly among children younger than five years and immune-compromised elderly ${ }^{13,14}$. These microorganisms are airborne transmitted particularly in healthcare settings (HCAP, healthcare-associated pneumonia) and at community level in crow ded areas (CA P, community acquired pneumonia $)^{15}$. Since airborne infections are frequent in dental healthcare settings, but data are lacking, the fourth study sought to investigate $\mathrm{S}$. pneumoniae carriage rate among adolescent dental patients ${ }^{16}$. Carriage rate is important to estimate whether pneumococci are frequently spread in the dental environment and transmission-based precautions are needed, including vaccination against pneumococci and, perhaps, influenza (most HCAP-CAP cases are consequent to the development of this disease) ${ }^{17}$. This paper reported a carriage rate as high as $11.6 \%$ and built a probabilistic model to individuate patients' profile allegedly associated with pneumococci carriage. Recent RTIs and routine exposure to passive smoking resulted in $58 \%$ probability of pneumococci carriage. Thus, a simple anamnesis could be enough to decide whether to implement transmissionbased precautions or not.

\section{H ow does Staphylococcus aureus spread in the dental environment?}

M ethicillin-resistant Staphylococcus aureus (MRSA) is the major responsible for healthcare-associated infections (HAIs) in the world. MRSA infections are estimated to affect more than 150,000 patients in EU and al most 400,000 in US annually, resulting in attributable extra costs to the heal thcare systems of $€ 380$ million in EU and $\$ 14.5$ billion in US. The risk for serious MRSA infections in dentistry is probably low. Nevertheless, staphylococci may spread in the dental environment and infections may occur among DHWs and patients. The main elements of transmission are DHWs, who could be carriers or vectors, and clinical contact surfaces contaminated by other carriers previously treated $^{18}$. The fifth reported study investigated staphylococci detected on GDPs' gloved working hands, soon after dental therapy, and on clinical contact surfaces, namely, the trays of the dental units ${ }^{19}$. Staphylococci detection rates were high $(41 \%$ and $57 \%$ on trays and gloves, respectively), the majority of them were coagulase-negative (CNS). S. aureus was detected in $5 \%$ of samples, while M R SA in $1.5 \%$ and methicillin-resistant CNS in $2 \%$ of samples. Very interestingly, whenever these microorganisms were detected on trays and gloves at the same time, they showed the same antibiotic profile, thus suggesting that the contaminant Staphylococcus strain was the same. In these episodes, GDPs did not result oral/nasal M RSA carriers.

This study suggested that patients could be a source of M RSA and GDPs could act as vectors, with their gloved working hands contaminated by these microorganisms. This study was made before infection control measures were implemented by GDPs, therefore, it was not 
possible to assess the environmental contamination remaining after decolonization. However, it stresses the necessity to adopt environmental infection control protocols.

\section{Is there a risk for infection associated with the use of finger rings?}

Transmission of pathogens through the hands of healthcare workers is demonstrated, particularly in intensive care units where immune-deficient patients are hospitalized and multiple resistant microorganisms are frequently detected. For this reason, most scientific and professional organizations recommend accurate hand hygiene. The same recommendation exists for DHWs, although there are no demonstrated cases of infection transmission attributable to imperfect hand hygiene ${ }^{20}$. One aspect associated with hand hygiene is the use of finger rings, because they are responsible for less accurate hand hygiene and for glove donning during wearing with consequent crosscontamination between heal thcare workers and patients. According to a Cochrane systematic review there is insufficient evidence in support of the hypothesis that removal of finger rings by healthcare workers may help prevent surgical infections ${ }^{21}$. However, a plethora of studies reported that hands of healthcare workers with finger rings yield a high level of hand contamination.

The aim of the sixth reported study was to compare the level and quality of hand contamination in a sample of dental hygienists with and without rings ${ }^{22}$. Potentially pathogenic bacteria were detected twice more frequently in ring wearers than in non-wearers, while fungi were detected almost five times more frequently. These data support the hypothesis that the use of rings beneath the gloves is responsible for high likelihood to harbour potential pathogens. Therefore, ring removal before starting the working session would be advisable, although it is not clear whether this measure is per se enough to help prevent infection transmission.

\section{Which is the most effective method to decolonize clinical contact surfaces?}

Infection transmission in dental healthcare settings is generally due to cross-contamination between DHWs and patients, which could be direct or mediated by dental equipment. Handtouch surfaces, also known as clinical contact surfaces, could be sometimes involved in transmission patterns, as suggested by cases of M RSA infection 18 and the only reported case of patient-to-patient transmission of hepatitis $B$ in dentistry ${ }^{23}$. Therefore, guidelines for infection control in dentistry must consider protocols for clinical contact surface decolonization. The proposed protocols include the use of disposal barriers that must be changed between patients and the use of disinfectants with tuberculocidal activity. Both methods have shortcomings: costs and environmental impact for barriers, costs and toxicity for disinfection. For this reason, cleaning could be an alternative solution on condition that it is effective enough to control for microbial contamination. Previous data on dental chairs artificially contaminated with MRSA reported a similar decontamination power of barriers, disinfection and cleaning ${ }^{24}$. The present study, made on cultivable flora which naturally contaminated the dental chair after endodontic or conservative therapy, found that cleaning with commercial soap and cleaning plus disinfection with hypochlorite had the same decontamination activity ${ }^{25}$, thus suggesting that accurate cleaning could be recommended for between-patient clinical contact surface decolonization.

\section{What is the difference between vaccination and immunity?}

Observational studies made around the $H B V$ vaccination era, showed that $H B V$ infection was an important occupational risk for DHWs and a serious risk for patients and that HBV immunity, along with the application of standard precautions, are the cornerstones of HBV control in dental healthcare settings 20,26 . Vaccination implies that serum antibody level against $\mathrm{HBV}$ surface antigen ( $\mathrm{HBs} \mathrm{Ag}$ ) is high enough to provide protection against HBV in- 
fection. However, this level tends to decrease with time and booster doses are periodically necessary. In addition, there are some non-responders who require additional vaccine doses. The aim of the eighth study was to investigate the potential discrepancies between vaccination and immunity among GDPs${ }^{27}$. The A uthors reported that almost $90 \%$ GDPs were vaccinated in the last 10 years, but $5 \%$ of them were not immune. The occupational HBV risk in these subjects is consequently high, as well as the risk for HBV transmission to patients, since infected DHWs are the main source of transmission in dental healthcare settings and there is only one reported case of transmission between patients $\underline{23}$.

\section{Conclusion - An effective infection control in dentistry}

There are several steps which lead to an effective infection control in dental healthcare settings. As previously noted, the first of them is an efficient surveillance system of infections acquired in dental healthcare settings ${ }^{28}$.
The second step is the specificity of scientific research. Many recommendations for infection control are gathered from healthcare settings, such as intensive care units or institutional long-term care settings, which have different characteristics than the dental healthcare settings. Recommendations which are useful and effective in these settings are not necessarily as useful and effective in dentistry.

The third step is that guidelines must be applied by DHWs, who, therefore, must be involved in guideline design and in recommendation release. There are too many examples of guidelines which did not reach a high degree of consensus among healthcare workers and, therefore, were only formally applied or even unapplied at all. Guidelines are more likely to be implemented if they are accepted and not imposed 4 .

The fourth and final step is that infection control guidelines must be transnational, as an increasing number of people seek for dental care outside the confines of their countries and, therefore, it would be desirable that the quality of dental care and the level of patient safety between different countries are comparable. 


\section{LITERATURA / REFERENCES}

1. Centers for Disease Control (CDC). Updatetransmission of HIV infection during an invasive dental procedure-Florida. MMWR Morb Mortal Wkly Rep 1991;40(23):377-81.

2. Rimland D, Parkin WE, Miller GB Jr, Schrack WD. Hepatitis B outbreak traced to an oral surgeon. N Engl J Med. 1977 A pr 28;296(17):953-8.

3. Shaw FE Jr, Barrett $C L, H a m m ~ R$ et al. Lethal outbreak of hepatitis $B$ in a dental practice. JAMA 1986;255(23):3260-4.

4. Petti S. Why guidelines for early childhood caries prevention could be ineffective amongst children at high risk. J Dent 2010;38(12):946-55.

5. Petti $S$, Polimeni $A$. The rationale of guidelines for infection control in dentistry: precautionary principle or acceptable risk? Infect Control Hosp Epidemiol 2010;31(12):1308-10.

6. M essano GA, Sofan AAA, Petti S. Quality of air and water in dental heal thcare settings during professional toothcleaning. A cta Stomatol Naissi 2013; 29(67): 123035; doi: 10.5937/asn1367230M .

7. Petti S, Moroni C, Messano GA, Polimeni A. Detection of oral streptococci in dental unit water lines after therapy with air turbine handpiece: biological fluid retraction more frequent than expected. Future Microbiol 2013;8(3):413-21.

8. Messano GA, Masood M, Palermo P, Petti S. Predictors of Legionella occurrence in dental unit waterlines of a highly colonized dental hospital. A cta Stomatol Naissi 2013; 29(67): 1236-41; doi: 10.5937/ asn1367236M .

9. Richeldi L, Ewer K, L osi M , Roversi P, Fabbri L M, Lalvani $A$. Repeated tuberculin testing does not induce false positive ELISPOT results. Thorax 2006;61(2):180.

10. Chihota VN, Nyazema NZ, Mashingaidze S $M$ utandiro B. TB infection: an exploratory study of BCG protective properties and the possibleroleof environmental mycobacteria. Cent A fr J M ed 1998;44(6):145-8.

11. Pankhurst CL, Coulter WA. Do contaminated dental unit waterlines pose a risk of infection? J Dent 2007;35(9): 712-20.

12. Messano GA, Masood M, Palermo P, Petti S. Prevalence of reactive tuberculin skin test in dental healthcare workers and students. A cta Stomatol Naissi 2013; 29(67): 1242-48; doi: 10.5937/asn1367242M.

13 O'Brien KL, Wolfson LJ, Watt JP et al. Burden of disease caused by Streptococcus pneumoniae in children younger than 5 years: global estimates. Lancet 2009;374(9693):893-902.

14 Wroe PC, Finkelstein JA, Ray GT et al Aging population and future burden of pneumococcal pneumonia in the U nited States. J Infect Dis. 2012 M ay 15;205(10):1589-92.
15. A merican Thoracic Society, Infectious Diseases Society of A merica. Guidelines for the management of adults with hospital-acquired, ventilator-associated, and healthcare-associated pneumonia. A m J Respir Crit Care M ed 2005;171(4):388-416.

16. Petti S, Sofan A A A, M essano GA. Streptococcus pneumoniae carriage rate in heal thy preadolescent dental patients. Acta Stomatol Naissi 2013; 29(67): 1249-54; doi: 10.5937/asn1367249P.

17. Harte JA. Standard and transmission-based precautions: an update for dentistry. J Am Dent Assoc 2010;141(5):572-81.

18. Petti S, Polimeni A. Risk of methicillin-resistant Staphylococcus aureus transmission in the dental healthcare setting: a narrative review. Infect Control Hosp Epidemiol 2011;32(11):1109-15.

19. Messano GA, De Bono V, Architrave $R$, Petti S. Environmental and gloves' contamination by staphylococci in dental healthcare settings. Acta Stomatol Naissi 2013; 29(67): 1255-59; doi: 10.5937/ asn1367255M

20. Kohn WG, Collins AS, Cleveland JL et al. Guidelines for infection control in dental health-care settings, 2003. MMWR Recomm Rep 2003;52(RR17):1-61.

21. A rrowsmith VA, Taylor R. Removal of nail polish and finger rings to prevent surgical infection. Cochrane Database Syst Rev 2012;5:CD 003325.

22. M essano GA. B acterial and fungal contamination of dental hygienists' hands with and without finger rings. A cta Stomatol Naissi 2013; 29(67): 1260-64; doi: 10.5937/asn1367260M .

23. Redd JT, Baumbach J, Kohn W, Nainan O, Khristova M, Williams I. Patient-to-patient transmission of hepatitis B virus associated with oral surgery. J Infect Dis 2007;195(9):1311-4.

24. Petti S, Polimeni A, Dancer SJ. Effect of disposable barriers, disinfection, and cleaning on controlling methicillin-resistant Staphylococcus aureus environmental contamination. A m J Infect Control 2013. doi: 10.1016/j.ajic.2012.09.021.

25. Petti S, Messano GA, Polimeni A, Dancer S]. Effect of cleaning and disinfection on naturally contaminated clinical contact surfaces. A cta Stomatol N aissi 2013; 29(67): 1265-72; doi: 10.5937/asn1367265P.

26. Petti S, Messano GA, Polimeni A. Dentists' awareness toward vaccine preventable diseases. Vaccine 2011;29(45):8108-12.

27. Petti S, M essano GA, Scully C. A ntibody level and immunity against $H$ epatitis $B$ virus infection among general dental practitioners. A cta Stomatol Naissi 2013; 29(67): 1273-78; doi: 10.5937/asn1367273P.

28. Laheij A M, K istler J O, B elibasakis GN, V älimaa $\mathrm{H}$, de Soet JJ; European Oral Microbiology Workshop (EOMW) 2011. Healthcare-associated viral and bacterial infections in dentistry. J Oral Microbiol 2012;4. doi: 10.3402/jom.v4i0.17659. 